\title{
Transient Receptor Potential Canonical 4 Channel in Interstitial Cells of Cajal as a Target for Control of Gastrointestinal Motility
}

TO THE EDITOR: There are 2 major roles attributed to interstitial cells of Cajal (ICC): (1) providing pacemaker activity to smooth muscle and (2) transmission of neural inputs to smooth muscle. ${ }^{1}$ Therefore, ICC together with neuron and smooth muscle cells constitute the phasic contractile activity and propagation within smooth muscle layers. This activity likes an orchestra by 3 type of cells, synchronizing the periodic rhythmicity.

The underlying synchronization is intercellular communication by which ICC bridging an action from neuron to smooth muscle, and transducing the mechanosensitivity from smooth muscle to neuron ie, regulates or alternates the excitation and relaxation. A study published in Journal of Neurogastroenterology and Motility entitled "Transient receptor potential canonical 4 and 5 channel antagonist ML204 depolarized pacemaker potentials of interstitial cells of Cajal" by Lee et $\mathrm{al}^{2}$ reveals that such rhythmicity is regulated through transient receptor potential canonical (TRPC) 4/5 channel operated $\mathrm{Ca}$ oscillation or mobilization to set up pacemaker potential in ICC.

There are several new findings in this study: (1) TRPC4 expression assayed by reverse transcription polymerase chain reaction suggests it is one of ICC properties as shown in Figure 1. (2) The rhythmically spontaneous intracellular calcium ions $\left[\mathrm{Ca}^{2+}\right] \mathrm{i}$ oscillations frequency is regulated by TRPC4/5 blocker as seen in Figure 2A, indicating one of the targets onto pacemaker activity. (3) Any ionic conductance through either non-selective cation channels (NSCC) or $\mathrm{Ca}^{2+}$-activated $\mathrm{Cl}$ - channels for pacemaker activity must be driven by $\left[\mathrm{Ca}^{2+}\right]$ i oscillation. None of the selective channel inhibitors are capable of direct blockage of the channel or ionic conductance. The fact is that all these inhibitors altering pacemaker activities are due to their changing membrane potential of ICC. As seen in Figure 2B, anoctamin did not abolish the pacemaker activity, and the other inhibitors respectively targeting on NSCC, anoctamin 1, ether-a-go-go-related gene potassium channel, and TRPC4

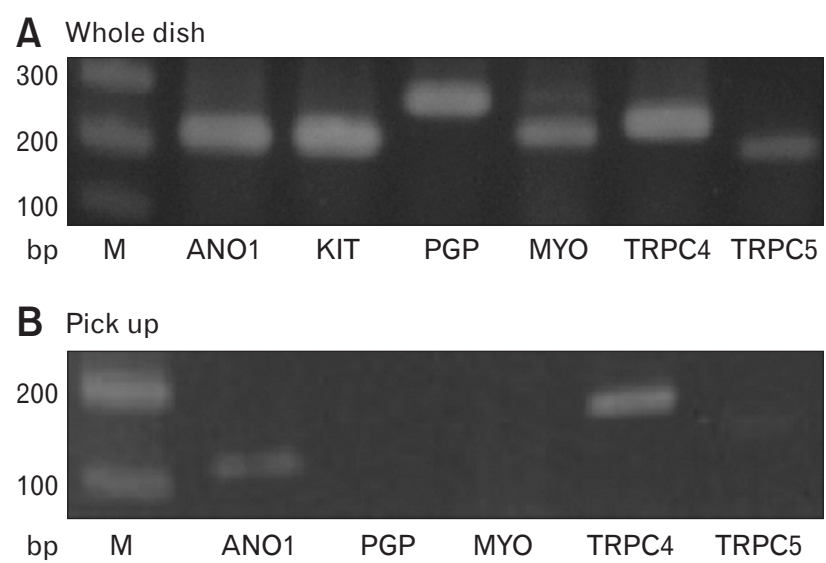

Figure 1. Transient Receptor Potential Canonical 4 and 5 (TRPC4/5) mRNA expression was obtained respectively from heterogeneous muscle layer dissociated cells and single interstitial cells of Cajal (ICC). (A) Heterogeneous muscle layer dissociated cells were mainly included neurons, smooth muscles, and ICC that were made in primary culture. Reverse transcription polymerase chain reaction (RT-PCR) screened the markers of neuron by proton gene product 9.5 (PGP), muscle by myosin (MYO), ICC by anoctamin 1 (ANO1), and c-Kit. Note that those markers are all strongly expressed in corresponding sizes, and TRPC4 strongly whilst TRPC5 weakly displayed. (B) Single ICC picked up by patch pipette suction was screened by RTPCR. Obviously, there is only ANO1 expressed, whilst there is no dimer in either neuron or muscle cells. TRPC4 transcript here can be only associated with ICC. Adapted from Lee et al. ${ }^{2}$ 
A
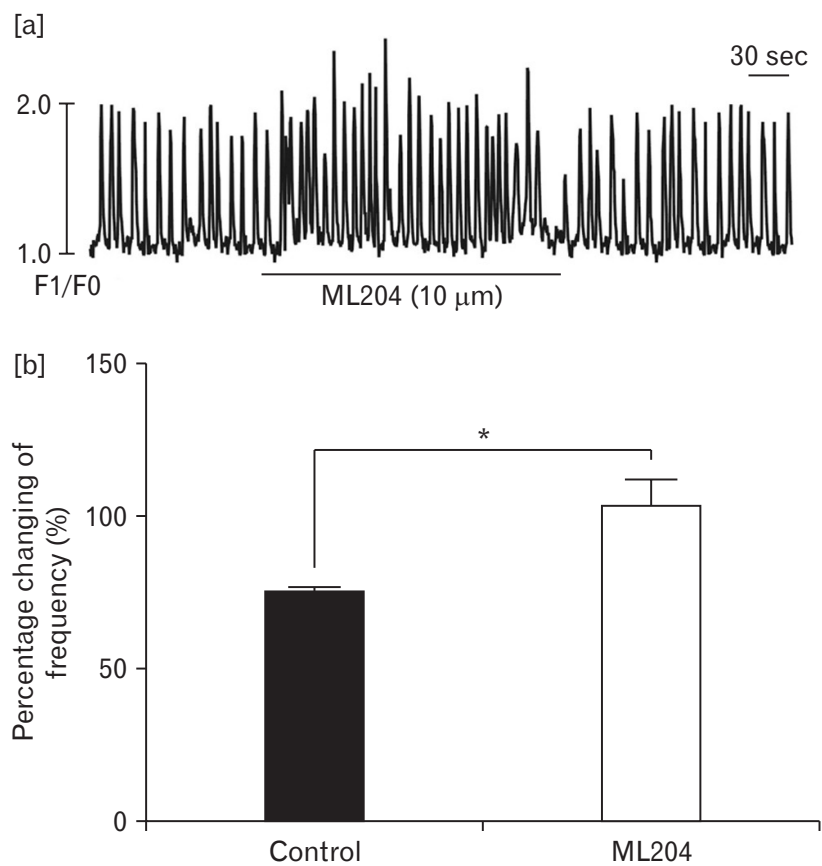

B

[a]

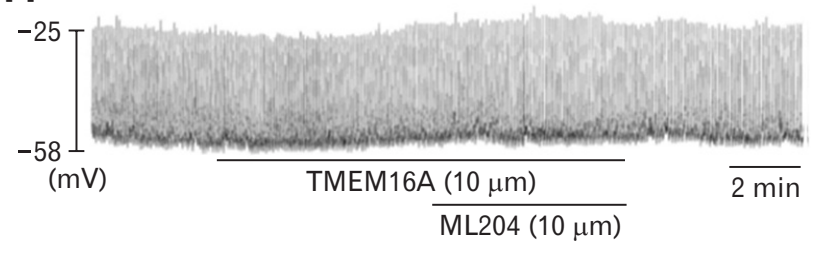

[b]

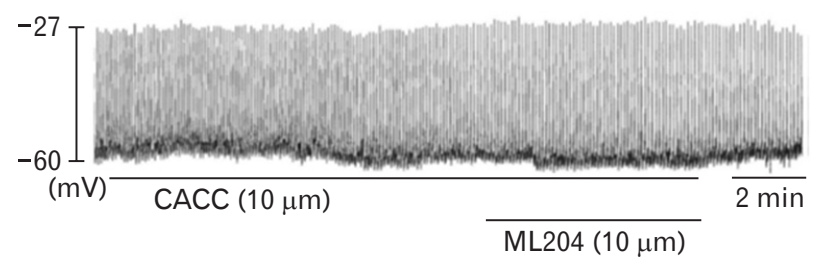

[c]

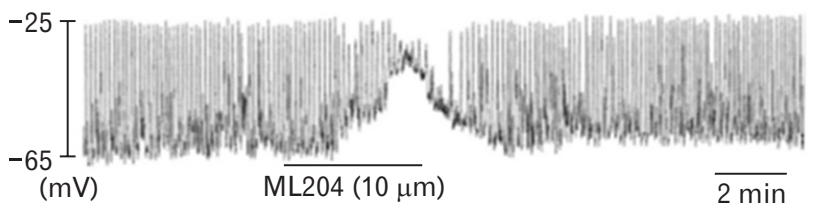

Figure 2. Transient Receptor Potential Canonical 4 (TRPC4) functional expression in interstitial cells of Cajal (ICC) was examined respectively by calcium (Ca)-imaging and pacemaker activity. (A) ICC intracellular $\mathrm{Ca}^{2+}\left[\mathrm{Ca}^{2+}\right] \mathrm{i}$ imaging was loaded with Fluo-4 acetoxymethyl ester that displayed the spontaneously biophysical oscillation, and the oscillating frequency was increased by TRPC4 inhibitor (ML204). [a] A trace of the spontaneous $\left[\mathrm{Ca}^{2+}\right]$ i oscillations before and after application of ML204. In general, all activity of the trace was featured with rhythmicity, consistent to the pacemaker activity in ICC. Here, TRPC4 inhibitor obviously elevated the baseline concentration and increased Ca oscillation frequency. $\mathrm{F} 1 / \mathrm{F}$ 0, relative alterations of $\left[\mathrm{Ca}^{2+}\right] \mathrm{i}$ fluorescence emission. [b] Histogram summarizes the frequency that was about $25 \%$ increased due to ML204 application. Bars represent mean \pm SE values ( $n=6$ per group). ${ }^{*} P<0.05$. (B) ICC pacemaker activity recorded from patch clamp was not obviously affected anoctamin 1 (ANO1) blocker or $\mathrm{Ca}^{2+}$-activated $\mathrm{Cl}$ - channel (CACC) blocker, but those blockers can prevent the effect from ML204 that greatly depolarize the membrane potential and consequently decreased pacemaker activity. [a] A trace recorded from a single ICC exhibiting pacemaker activity was not inhibited by transmembrane member 16A (TMEM16A), a specific blocker (ANO1). Addition of ML204 in presence of TMEM16A did not change as much as it is compared to [c] alone. [b] Similar effect of TMEM16A from CACC also did not change the pacemaker activity but prevented the depolarization from M204. [c] ML204 $10 \mu \mathrm{M}$ drastically depolarized the membrane potential and inhibited pacemaker activity but it was reversible after washing out. Adapted from Lee et al. ${ }^{2}$

channel are because of their depolarizing membrane potential. ${ }^{3-6}$

Lee et $\mathrm{al}^{2}$ examined TRPC4 in ICC by mRNA transcripts, $\mathrm{Ca}$ fluorescent imaging and electrophysiological assays. They demonstrated that TRPC4 channel is one of properties in ICC and actively participated in pacemaker activity by possibly different mechanisms, in which TRPC4 in ICC controls of intracellular $\mathrm{Ca}$ concentration, or balances the dynamic processes of neurotransmitter and mechanosensitivity in setting up membrane potential or initiating the excitability of the ICC. Therefore, regulation of the second messenger system through TRPC4 is highly susceptible in modulating ICC pacemakers, therefore, the TRPC4 channel is a target for control of gastrointestinal motility.
Zengzi Zhou, ${ }^{1}$ Meng Xia, ${ }^{2}$ and Jianlin $\mathrm{Lv}^{3 *}$ ${ }^{1}$ The 3rd Xiangya Hospital, Central South University, Changsha, Hunan, China; ${ }^{2}$ Guangxi university of Chinese Medicine, Nanning, Guangxi, China; and ${ }^{3}$ The First Affiliated Hospital of Guangxi University of Chinese Medicine, Nanning, Guangxi, China

1. Sarna SK. Are interstitial cells of Cajal plurifunction cells in the gut? Am J Physiol Gastrointest Liver Physiol 2008;294:G372-G390.

2. Lee JH, Wu WH, Huang XY, Jun JY, Choi SC. Transient Receptor Potential Canonical 4 and 5 channel antagonist ML204 depolarized pacemaker potentials of interstitial cells of Cajal. J Neurogastroenterol Motil 2020;26:521-528.

3. Koh SD, Sanders KM, Ward SM. Spontaneous electrical rhythmicity in cultured interstitial cells of Cajal from the murine small intestine. J Physiol 1998;513(Pt 1):203-213. 
4. Gomez-Pinilla PJ, Gibbons SJ, Bardsley MR, et al. Ano1 is a selective marker of interstitial cells of Cajal in the human and mouse gastrointestinal tract. Am J Physiol Gastrointest Liver Physiol 2009;296:G1370G1381.

5. Sanders KM, Zhu MH, Britton F, Koh SD, Ward SM. Anoctamins and gastrointestinal smooth muscle excitability. Exp Physiol 2012;97:200206.

6. Zhu Y, Golden CM, Ye J, Wang XY, Akbarali HI, Huizinga JD. ERG $\mathrm{K}^{+}$currents regulate pacemaker activity in ICC. Am J Physiol Gastroin- test Liver Physiol 2003;285:G1249-G1258.

Financial support: This work was supported by The first Batch of Qihuang High-level Talent Team Training Projects of Guangxi University of Chinese Medicine (Award No. 2018001).

Conflicts of interest: None.

Author contributions: Zengzi Zhou and Meng Xia are equal in writing manuscript, and Jianlin Lv oversights the study and revising. 